\title{
Prevalence of Multimorbidity in Adults with Cancer, and Associated Health Service Utilization in Ontario, Canada: A Population-based Retrospective Cohort Study
}

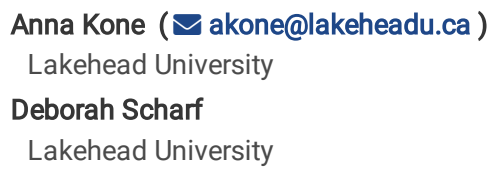

Version of Record: A version of this preprint was published at BMC Cancer on April 14th, 2021. See the published version at https://doi.org/10.1186/s12885021-08102-1. 


\section{Abstract}

Background: The majority of people with cancer have at least one other chronic health condition. With each additional chronic disease, the complexity of their care increases, as does the potential for negative outcomes including premature death. In this paper, we describe cancer patients' clinical complexity (i.e., multimorbidity; $\mathrm{MMB}$ ) in order to inform strategic efforts to improve care and outcomes for people with cancer of all types and commonly occurring chronic diseases.

Methods: We conducted a population-based, retrospective cohort study of adults diagnosed with cancer between 2003 and 2013 ( $N=601,331$ ) identified in Ontario, Canada healthcare administrative data. During a five to 15-year follow-up period (through March 2018), we identified up to 16 co-occurring conditions and patient outcomes including health service utilization and death.

Results: MMB was extremely common, affecting more than $91 \%$ of people with cancer. Nearly one quarter (23\%) of the population had five or more cooccurring conditions. While we saw no differences in MMB between sexes, MMB prevalence and level increased with age. MMB prevalence and type of cooccurring conditions also varied by cancer type. Overall, MMB was associated with higher rates of health service utilization and mortality, regardless of other patient characteristics, and specific conditions differentially impacted these rates.

Conclusions: People with cancer are likely to have at least one other chronic medical condition and the presence of MMB negatively affects health service utilization and risk of premature death. These finds can help motivate and inform health system advances to improve care quality and outcomes for people with cancer and MMB due to commonly occurring chronic conditions.

\section{Introduction}

Multimorbidity (MMB), defined as the co-occurrence of multiple chronic conditions, is a public health crisis, challenging healthcare systems and affected individuals and their families (1-5). The likelihood of having multiple chronic health conditions is particularly high among people with cancer (4, 6-10). In Ontario, Canada, more than $75 \%$ of people with cancer have at least one of 16 other prevalent chronic conditions (4). Rates of MMB among cancer patients, however, vary by cancer type $(6,11,12)$. Similarly, the chronic condition(s) that co-occur with cancer also vary by cancer type and can have different impacts on patient outcomes, including death $(11,13,14)$.

MMB impacts people with cancer directly through an increased physiological burden of disease and/or indirectly through healthcare factors related to treatment decision-making and the increased complexity of care (15). For example, MMB can complicate and delay cancer and other diagnoses, increasing treatment complexity, risk of polypharmacy, and/or other redundancies in care (16-20). Critically, cancer patients with MMB are less likely to be offered active or curative therapies (21-23), despite growing evidence that such treatments are tolerated and effective for some patients with cancer MMB (24-26). People with cancer and MMB likely also experience increased burden from managing multiple appointments and care regimens, which may result in confusion, stress, difficulty adhering to treatment, and poor quality of life.

Another critical challenge to effectively treating and improving outcomes for people with cancer and other chronic conditions is that most care plans follow a single disease-oriented approach $(10,18,27,28)$. In such single-disease models, the management of co-occurring conditions often takes a backseat to cancer even when coordinated management of chronic conditions could help improve patient outcomes and quality of life $(29,30)$. Well-documented gaps in clinical guidelines and care management frameworks or resources demonstrate the need for intentional integration and coordination of care for patients with MMB whose care frequently spans siloed specialized and primary care settings $(2,31-33)$, including clear direction to provider who may lack clarity about their roles and responsibilities in managing complex cancer patients' care (34-36).

In order to improve care and outcomes for people with cancer and $\mathrm{MMB}$, health care systems must recognize and reorganize care delivery in order to provide whole-person care. However, few large epidemiological studies have examined the overall extent of MMB among people with cancer of all types to provide guidance about how care should be reorganized. Similarly, researchers have not broadly documented the potential impact of cancer MMB on health service utilization at the general population-level in order to effectively motivate care delivery reform. In this paper, we address these research gaps by describing the co-occurrence of cancer of any type with 16 common chronic diseases in the large province of Ontario, Canada, as well as the level of MMB associated with patients' health service utilization in a population-based, retrospective cohort study. In a second, companion paper using this same data set, we examine which conditions are most likely to co-occur with cancer and how specific disease clusters impact patients' outcomes (Koné et al., under review).

\section{Methods}

\section{Study design and data sources}

We performed a retrospective cohort study of people diagnosed with cancer in Ontario, Canada using population-based healthcare administrative data. The province of Ontario provides universal coverage of physician visits and hospital services for virtually all residents. This study population includes all individuals 18 years and older living in Ontario with a valid health card, who received a cancer diagnosis between April 1, 2003 and March $31,2013$. Individuals were then followed-up through March 31, 2018 in order to track the occurrence of 16 co-occurring chronic health conditions and health service utilization (HSU).

As in our previous work (4), data came from the Institute for Clinical Evaluative Sciences (ICES) linked data sets including information from: The Registered Persons Database (RPDB), the Canadian Institute for Health Information's Discharge Abstract Database (DAD), the Ontario Health Insurance Plan (OHIP) claims database, the Ontario Drug Benefits (ODB), and the National Ambulatory Care Reporting System (NACRS). ICES used unique encoded identifiers to 
create the study population, to identify occurrence of conditions, and measure health service utilization. ICES made the cohort available on the secure (IDAVE) website for remote analyses by the researchers. The Lakehead University Research Ethics Board reviewed and approved this research.

\section{Measures}

Multimorbidity. We defined MMB as the co-occurrence of any of 16 conditions specified below, co-occurring with any cancer diagnosis, similar to previous research $(4,37,38)$.

Multimorbidity level. We defined and grouped individuals by level of MMB as defined by the presence of 1, 2, 3, 4, or 5 or more conditions, as compared to cancer only.

Co-occurring conditions. We identified the presence of 16 chronic conditions using hospital discharge (DAD), physician billings (OHIP) and drug prescription (ODB) data. These conditions included: acute myocardial infarction (AMI), asthma, cancer, cardiac arrhythmia, chronic obstructive pulmonary disease (COPD), congestive heart failure (CHF), chronic coronary syndrome, dementia, diabetes, hypertension (HT), non-psychotic mood and anxiety disorders, other mental illnesses, osteoarthritis, osteoporosis, renal failure, rheumatoid arthritis, and stroke.

Cancer. We used cancer ICD-0-3 topography and histology codes and stage indicators available from the Ontario Cancer Registry (OCR). Cancer type was then defined according to SEER program for site groups

Health services utilization. We included several measures of health service utilization including number of primary care (PC) visits, emergency department (ED) visits, and hospital admissions. To account for different lengths of follow-up and/or death, we defined HSU in two ways for individuals with more 30 days of follow-up post cancer diagnosis: 1) by counts during the first-year post cancer diagnosis, and 2) average person-year counts for entire follow-up.

Survival. Survival was defined as time to all-cause death during follow-up, using death date from the RPDB. Rate of mortality was also calculated within one year, 5 years and overall during follow-up for those who survived at least 30 days post cancer diagnosis.

Demographics. Basic demographic variables including age, sex, and geographic region of residence were obtained from the RPDB.

\section{Analyses}

We used descriptive statistics to characterize population demographics, common cancer sites, and MMB level. We then used bivariate and age-stratified analyses to assess cancer complexity relative to participant demographics, cancer type, HSU, and survival. Finally, we used negative binomial and logistic regressions to assess the adjusted impact of increasing cancer complexity on health service utilization and survival within the first year, using pre-existing MMB levels (i.e. up to 30 days after cancer diagnosis) and controlling for age, sex, cancer types and stages. We used SAS v. 10 to conduct all analyses.

\section{Results}

Our study population included 601,331 adults diagnosed with cancer between 2003 and $2013 ; 49 \%$ were females and $55.7 \%$ aged 65 years or older. The most common types of cancer identified in the sample were (in descending order): Prostate, breast, colon/rectum, and lung/bronchus (Table 1). 


\begin{tabular}{|c|c|}
\hline Characteristics & $\mathbf{N}(\%)$ \\
\hline Female Sex & $294,490(49)$ \\
\hline Age group & $49,362(8.2)$ \\
\hline $45-64$ & $217,229(36.1)$ \\
\hline $65+$ & $334,740(55.7)$ \\
\hline Rural residency & $85,547(14.2)$ \\
\hline Central & $190,015(31.6)$ \\
\hline Central east & $94,127(15.7)$ \\
\hline East & $83,660(13.9)$ \\
\hline North & $45,937(7.6)$ \\
\hline South & $104,718(17.4)$ \\
\hline Southwest & $82,592(13.7)$ \\
\hline Brain and Other Nervous System & $8,553(1.4)$ \\
\hline Breast & $81,553(13.6)$ \\
\hline Cervix Uteri & $5,197(0.9)$ \\
\hline Colon and Rectum & $71,754(11.9)$ \\
\hline Digestive System, except Colon & $49,621(8.3)$ \\
\hline Endocrine System & $22,564(3.8)$ \\
\hline Female Genital System, except & $30,983(5.2)$ \\
\hline Leukemia & $16,996(2.8)$ \\
\hline Lung and Bronchus & $75,619(12.6)$ \\
\hline Lymphoma & $28,437(4.7)$ \\
\hline Myeloma & $8,487(1.4)$ \\
\hline Oral Cavity and Pharynx & $13,715(2.3)$ \\
\hline Other & $39,691(6.6)$ \\
\hline Prostate & $87,377(14.5)$ \\
\hline Skin excluding Basal and Squam & $23,952(4)$ \\
\hline Urinary System & $36,832(6.1)$ \\
\hline No additional condition & $52,083(8.7)$ \\
\hline 1 & $97,478(16.2)$ \\
\hline 2 & $118,310(19.7)$ \\
\hline 3 & $109,166(18.2)$ \\
\hline 4 & $85,077(14.1)$ \\
\hline 5 or more conditions & $139,217(23.2)$ \\
\hline Follow-up in years: Average (SD); range & $5.8(4.5) ; 0^{*}-15$ \\
\hline \multicolumn{2}{|l|}{ Health service use within the first year (follow-up >=30 days**) } \\
\hline Primary care visit: at least one & $519,752(92.2)$ \\
\hline Primary care visit: High use (90th pct, i.e. $>=15$ ) & $63,835(11.4)$ \\
\hline ED visit: at least one & $228,280(40.7)$ \\
\hline ED visit: High use (90th pct, i.e. >3) & $57,239(10.2)$ \\
\hline Hospital admission: at least one & $325,513(58.1)$ \\
\hline Hospital admission: High use (90th pct, i.e. >=2) & $127,976(22.8)$ \\
\hline
\end{tabular}




\begin{tabular}{|c|c|c|}
\hline Characteristics & & $N(\%)$ \\
\hline Mortality (follow-up $>=30$ days) & Within first year & $107,378(19.2)$ \\
\hline Within five years & & $219,315(39.1)$ \\
\hline Overall, during follow-up & & $281,268(49.8)$ \\
\hline
\end{tabular}

\section{Cancer patients were likely to be complex, with high levels of MMB}

The majority of people with cancer (91.3\%) had one or more co-occurring chronic conditions; nearly one quarter (23.2\%) had five or more co-occurring conditions (Table 1). Complexity was similar between men and women, but more prevalent among those 65 or more years of age (Figures $1 \mathrm{~A} \& \mathrm{~B}$ ). MMB prevalence and levels varied across cancer types. For example, $9.4 \%$ of patients with cervix uteri cancer had five or more co-occurring conditions while $36.4 \%$ of people with myeloma had five or more co-occurring conditions (Figure 1C).

Most people (84\%) had MMB at the time of cancer diagnosis (before and up to 30 days after diagnosis). After cancer diagnosis, $45 \%$ of patients developed at least one additional condition during follow-up; rates of new chronic conditions after cancer diagnosis were similar (47\%) among those with no MMB at the time of cancer diagnosis. Overall, $22 \%$ of the complex population received all MMB diagnoses within the first year of cancer, and $70 \%$ received all of their diagnoses within 5 years (results not shown).

\section{Health service utilization increased with MMB level, regardless of patient characteristics}

Our design allowed us to follow individuals in the population for up to 15 years. During the follow-up period and, among those with at least 30 days of followup observation, $92 \%$ had at least one primary care visit, $41 \%$ had at least one ED visit, and $58 \%$ had at least one hospital admission within the first year (Table 1). Utilization rate during follow-up among those with at least one service visit was 6 primary care visits, 0.7 ED visit, and 0.5 hospitalization per year, respectively. The proportion of patients with high use (90th percentile) increased with increasing MMB. For example, of those with five or more conditions, $22 \%$ for PC visits, $14 \%$ for ED visits and $28 \%$ for hospital admissions were high users (data not shown).

HSU was the highest during the first year following cancer diagnosis. The number of PC visits, ED visits and hospitalizations increased with higher-levels of $\mathrm{MMB}$ in all age groups, in the first year and throughout the follow-up period (Figure 2).

The differences between MMB levels in terms of HSU were more substantial among younger adults, ages 18 to 44 years. In the adjusted models, increasing complexity was associated with higher rates of HSU. While there was no clear gradient related to PC visits, those with five or more conditions beside cancer exhibited $51 \%$ and $32 \%$ more ED visits and hospital admissions than those with cancer only (Figure 3 ). Individual co-occurring conditions had varying impacts on HSU; however, the effects of individual conditions appeared less, in the presence of higher levels of MMB (Figure 4).

\section{Cancer survival was associated with cancer type and age, and MMB had an incremental, independent negative impact}

The overall five-year survival rate among the study population followed for at least 30 days was $61 \%$, ranging from $18 \%$ to $95 \%$ by cancer type. The incremental impact of MMB, however, was consistent and negative for cancer types with similar prognoses. An exception was the limited impact of MMB on survival among people with lethal cancer types (Figure 5).

About half of the population died during follow-up and the mortality rate within one year was $19.2 \%$ (Table 1). Early death occurred mostly among those with lung/bronchus, digestive system (except colon) and brain and other nervous system cancers (data not shown). The risk of dying within the first year among individuals with between one and three co-occurring conditions was lower or similar to those with no condition prior to cancer diagnosis, whereas those with five or more conditions were $45 \%$ more likely to die, regardless of age, sex, cancer type or stage (Figure 3 ).

The impact of individual chronic conditions was largely different within and between levels of MMB. For example, the risk of death varied from 0.43 (osteoporosis) to 2.33 (renal disease) among those with one condition prior to cancer, and from 0.85 (hypertension) to 1.49 (CHF) among those with five or more conditions prior to cancer (Figure 4). Surprisingly, COPD decreased the risk of death by $17 \%$ when present alone before cancer, while increasing the risk of death by $37 \%$ when present with at least four other conditions.

Detailed results of the adjusted impact of MMB on HSU and mortality overall and by age group are in Appendices 1 and 2.

\section{Discussion}

This population-level study is among the first to examine the co-occurrence of cancer of all types with 16 other chronic conditions managed outside of the cancer care system. While research on MMB continues to grow, there are only few studies assessing the number of co-occurring conditions among people with 
cancer, and those studies have typically included only a few specific cancer sites $(7,8,11)$. By describing the extent of MMB within cancer of all types, we aim to inform Canadian and global efforts to improve quality, efficiency, and patient outcomes and experiences in complex (i.e., multimorbid) cancer care.

Overall, the prevalence of MMB in cancer patients is among the highest (4) (Kone et al 2021 accepted). Similar to previous studies (7, 11), we found that MMB was extremely common among people with cancer, reaching $91 \%$ in our population of patients diagnosed with cancer between 2003 and 2013 . Our data also showed that rates of MMB remained stable over time, indicating the ongoing need to address MMB effectively.

While patients are likely to exhibit multiple conditions before cancer, MMB continues to increase substantially after a cancer diagnosis. In our study we found that nearly half of all people with cancer were diagnosed with an additional chronic condition during follow-up. This is consistent with data from Leach and colleagues (7) in which cancer patients reported the emergence of approximately 1.9 new conditions after diagnosis of five common cancer types.

Our data is also consistent with previous studies showing no sex difference in MMB rates but increased rates and levels of MMB among older age groups ( 9 , 11). Notable from our study, however, are findings that cancer MMB were observed across all types, at any age of diagnosis, including high rates of cancer MMB of two or more conditions in young adults, ages 18-44 years. This suggests that MMB is not only an issue for older adults but a growing issue for adults of all ages $(4,39,40)$ (Kone et al 2021 accepted).

Cancer sites with the highest prevalence of MMB were myeloma, prostate, urinary system, lung/bronchus and Leukemia. For example, those with four or more conditions beside cancer represented a substantial proportion for all sites of cancer from $19 \%$ (cervical cancer) to $53 \%$ (myeloma). Similar to our study, Fowler et al (11) found that MMB was higher among people with lung cancer compared to the other four cancers included in their study, with $67 \%$ of lung cancer patients having one or more comorbidities. Different from Fowler and colleagues, however, is that our overall rates of MMB are higher. An important difference between our methods is that Fowler et al (11) considered 4 conditions instead of 16 included here. Other contextual factors, such as public health screening initiatives, may also be important for between-population and between-cancer rates of MMB. For example, in Ontario Canada, cervical cancer was among the sites with the lowest prevalence of MMB in our study ( $66 \%$ before cancer and $82 \%$ overall) but it is also the target of a province-wide screening initiative inplace for women as young as age 21 (41), who are less likely than older adults to have MMB.

We also analyzed the impact of MMB on multiple aspects of the healthcare utilization. While adequate access to primary care may contribute to better care management and patient outcomes, high use of ED and hospital services, and ultimately death, reflect poor patient health which may be aggravated by the presence of multiple conditions. Not surprisingly, both mortality and health service use were positively associated with increasing MMB levels, regardless of patients' age, sex, cancer type This population-level study is among the first to examine the co-occurrence of cancer of all types with 16 other chronic conditions managed outside of the cancer care system. While research on MMB continues to grow, there are only few studies assessing the number of cooccurring conditions among people with cancer, and those studies have typically included only a few specific cancer sites $(7,8,11)$. By describing the extent of MMB within cancer of all types, we aim to inform Canadian and global efforts to improve quality, efficiency, and patient outcomes and experiences in complex (i.e., multimorbid) cancer care.

The prevalence of MMB in cancer patients is extremely high (4) (Kone et al 2021 accepted). Similar to previous studies (7, 11), we found that MMB was nearly universal (91\%) among people with cancer diagnosed with cancer between 2003 and 2013. Our data also showed that rates of MMB remained stable over time, indicating that the need to address MMB effectively is long-standing and ongoing.

While most people (84\%) had MMB before cancer, MMB continues to increase after cancer diagnosis. In our study we found that nearly half of all people with cancer were diagnosed with an additional chronic condition during follow-up. This is consistent with data from Leach and colleagues (7) who found that patients reported the emergence of approximately 1.9 new conditions following a diagnosis of one of five common cancer types.

Our data is also consistent with previous studies showing no sex difference in MMB rates but increased rates and levels of MMB among older age groups (9, 11). Notable from our study is that we observed MMB across all cancer types, all ages of diagnosis, including high rates of cancer MMB of two or more conditions in young adults, ages $18-44$ years. This suggests that cancer MMB is not only an issue for older adults but for adults of all ages (4, 39, 40 ).

Cancer sites with the highest prevalence of MMB in our study were myeloma, prostate, urinary system, lung/bronchus and Leukemia. However, people with four or more comorbidities conditions were present among people with cancer of all sites, from 19\% (cervical cancer) to 53\% (myeloma). Similar to our study, for example, Fowler et al (11) found that MMB was highest among people with lung cancer compared to the other four cancers in their study, with $67 \%$ of lung cancer patients having one or more comorbidities. Different from Fowler and colleagues, however, is that our overall rates of MMB are higher. This is perhaps because they considered four possible co-morbid conditions instead of the 16 included here. Other contextual factors may affect between-population and between-cancer rates of MMB reported here and in Fowler et al (11). For example, in our Ontario, Canada, population, cervical cancer was among the sites with the lowest prevalence of $\mathrm{MMB}(66 \%$ before cancer and $82 \%$ overall) perhaps because it is also the target of a province-wide screening initiative in-place for women as young as age 21 (41), who are less likely than older adults to have MMB.

We also analyzed the impact of MMB on multiple aspects of the HSU. While adequate access to primary care may contribute to better care management and patient outcomes, high use of ED and hospital services, and ultimately death, reflect poor patient health which may be aggravated by the presence of multiple conditions. Not surprisingly, both mortality and health service use were positively associated with increasing MMB levels in our study, regardless of patients' age, sex, cancer type or stage. This is in line with previous research showing that higher MMB was associated with a higher risk of death or lower survival among patients with specific cancer types $(6,12,42)$. Legler et al (2011) also found a positive association between high patient Charlson Comorbidity Index and increased admission to ED, ICU and hospital (43). Unlike prior work, a unique contribution of this paper is that we examined the relationship between MMB level and HSU. In those analyses, we observed that increasing MMB level had a greater negative impact on HSU among younger people; the difference between cancer only and the highest level of MMB was on average 1.8 ED visits and 7.1 PC visits in young adults compared to 0.4 and 4.3 among people 65

Page 6/14 
years and older. The trend was opposite for mortality: increasing MMB was not as strongly linked to risk of death among younger adults. More research examining potential contributing factors, such as early screening and identification through regular health service use, for example, is needed to better understand cancer MMB in younger adults.

Lastly, our data showed that individual conditions had varied impact on patients' health outcomes, depending on the level of MMB. Conditions frequently identified as co-occurring with cancer in previous studies include hypertension, COPD, diabetes, CVD, CHF $(11,43)$. These conditions were also among the most frequent in our study population. The most common co-occurring conditions (hypertension, arthritis, anxiety, diabetes) often had the greatest impact on outcomes; however, other less prevalent conditions are also worth considering. Overall, the relationship between cancer, mental health (including substance use disorder) diagnoses, and HSU is in need of further study. Our data showed increased PC encounters among people with co-occurring anxiety disorder, which was also associated with fewer hospitalizations and a lower risk of death. While it well known that psychiatric conditions are in general a significant driver of HSU (e.g., (44)), conditions like anxiety that increase preventive contacts with the healthcare system may represent a protective form of MMB. Other conditions such as dementia or psychosis that limit patient capacity for self-care, in comparison, may increase higher-acuity service use, such as ED visits and hospitalizations, signaling the need for more intensive preventive care and/or illness self-management supports. Overall, these findings suggest that while $\mathrm{MMB}$ is important to understand overall, unique combinations of co-occurring conditions are likely to have differential effects on HSU and patient outcomes, and thus require further study.

Strengths and Limitations. This is the first population-based cohort study to examine the burden of MMB and its impact in the Ontario population of people with all types of cancer. It presents many strengths, including the size of the study population, extended follow-up of between five and 15 years, and the use of administrative data including multiple chronic conditions, including indicators of mental health. Because Ontario has universal health coverage, Ontario's health administrative data, provide robust, population-based estimations of cancer MMB (45) not available from self-report or other sources. In fact, with comprehensive health administrative data, estimates of MMB are more likely to be reliable and complete than patient self-report (46). In this study, we operationalized MMB through 16 high-burden chronic conditions, both prior to cancer and for five or more years following cancer diagnosis. Most of these chronic conditions were operationalized using validated algorithms; however, some conditions may not be adequately represented $(47,48)$. Namely, studies have found mixed results related to the under- or over-diagnosis of co-occurring conditions in cancer patients $(49,50)$. Though the number of conditions is adequate to assess overall MMB (51), another limitation is that other potentially relevant chronic conditions were not included which could have unique effects as individual diseases and/or as part of the effects of MMB level. Another limitation of this report is that the severity of non-cancer conditions is not considered in our assessment.

In addition to our main objective of describing MMB in cancer patients, we also aimed to advance the understanding of MMB on HSU and mortality. To do this, our analysis included only basic patient demographics and an indicator of cancer severity, which is sufficient to describe baseline impacts of MMB and develop hypotheses and rationale for further research. That said, we acknowledge that many other potentially confounding variables, such as socioeconomic status, could have potentially impacted our results and as such may be important in future work. There may also be some residual confounding regarding cancer stage because of the limitations in the staging data and the large proportion of missing information (presented as unknown); however, we believe that this approach is adequate to support the exploratory nature of this work. We also did not examine treatment approaches and quality of care that can play a crucial role in cancer outcomes.

\section{Conclusions \& Implications}

People with cancer are very likely to experience MMB over all and at very high levels, regardless of age and cancer type - including young adults whose risk for MMB may have been previously overlooked. The findings in this report can inform health system advances towards person-centered care as it describes the overall nature $\mathrm{MMB}$ for the whole range of cancer types. At the same time, our findings also show that there is wide variation in the impact of individual conditions within and between MMB levels, suggesting that it is crucial to assess the role of each condition within a MMB lens. This could for example, include routine assessment of chronic conditions that commonly co-occur with cancer and coordinated care pathways that reduce treatment burden and increase access to targeted supports (e.g., mental health; illness self-management) for patients likely to experience the need. More generally, however, our findings show that at higher levels of MMB, individual conditions have less of an impact on outcomes that MMB itself, again highlighting the need to improve how care for people with MMB is envisioned and put in-place. In the next paper, we describe the relationship between specific, observed clusters of cancer and chronic disease and their relationship to HSU and mortality risk to help guide next steps in advancing MMB care.

\section{Abbreviations}

CHF: Congestive heart failure

COPD: Chronic obstructive pulmonary disease

CVD: Cardiovascular disease

DAD: Discharge Abstract Database

ED: Emergency Department

HSU: Health services utilization

ICES: Institute for Clinical and Evaluative Sciences 
ICD-0-3: International Classification of Diseases for Oncology, 3rd version

MMB: multimorbidity

NACRS: National Ambulatory Care Reporting System

ODB: Ontario Drug Benefits

OHIP: Ontario Health Insurance Plan

PC: Primary Care

RPDB: Registered Persons Database

SEER: Surveillance, Epidemiology, and End Results

\section{Declarations}

\section{Ethics approval and consent to participate}

This research has been approved by the Research Ethics Board (IRB) at Lakehead University, Thunder Bay, Canada, as required by ICES' Privacy and Legal Office for external researchers. The need for consent to participate was waived by this Institutional Review Board (IRB). ICES is a prescribed entity under section 45 of Ontario's Personal Health Information Protection Act. Section 45 authorizes ICES to collect personal health information, without consent, for the purpose of analysis or compiling statistical information with respect to the management of, evaluation or monitoring of, the allocation of resources to or planning for all or part of the health system. This project was conducted under section 45, and approved by ICES' Privacy and Legal Office. All individual-level data were anonymized and only available to authorized researchers through a secure platform. REB approval from Lakehead University as well as ICES authorization are available upon request.

\section{Consent from participants}

The need for consent to participate was waived by Lakehead University Institutional Review Board (IRB), and according to ICES' rules. As a prescribed entity under PHIPA, ICES is authorized to collect personal health information from health organizations without consent for the purposes of evaluation and monitoring of Ontario's health system. ICES is prohibited, under its agreements with data providers, from contacting individuals whose information has been entrusted to ICES. This contractual obligation restricts any opportunity to seek individuals' consent for use of their information for research.

\section{Methods}

All methods were carried out in accordance with relevant guidelines and regulations, including data acquisition, cleaning, analyses and reporting of findings.

\section{Availability of data and materials}

The data that support the findings of this study are available from the Institute for Clinical and Evaluative Sciences (ICES), but restrictions apply to the availability of these data, which were used under the service agreement ICES DAS \# 2020-727, and so are not publicly available. Access may only be granted under specific criteria and confidentiality conditions; please see www.ices.on.ca/DAS. The contact person is Refik Saskin, Staff Scientist, Data \& Analytics Services ICES Central, (1) 416-480-4055.

\section{Competing interests}

The authors declare that they have no competing interests.

\section{Funding}

This work was supported by an Ontario Ministry of Health grant [MOHLTC Grant No. 06034]. The opinions and statements expressed herein are solely those of the authors and do not reflect those of the MOHLTC; no endorsement is intended or should be inferred.

\section{Authors' contributions}

APK conceptualized the study, analyzed and interpreted the data, and wrote the manuscript. DS conceptualized the study, interpreted the findings and was a major contributor in writing the manuscript. All authors read and approved the final manuscript. 


\section{Acknowledgements}

This study was supported by ICES, which is funded by an annual grant from the Ontario Ministry of Health and Long-Term Care (MOHLTC). The researchers contracted ICES Data \& Analytic Services (DAS) and used de-identified data from the ICES Data Repository. The analyses, conclusions, opinions and statements expressed herein are solely those of the authors and do not reflect those of the funding or data sources; no endorsement is intended or should be inferred.

The authors would like to particularly acknowledge the analytical support from Yu Qing [Chris] Bai, Associate Analyst at ICES UofT.

\section{References}

1. Barnett K, Mercer SW, Norbury M, Watt G, Wyke S, Guthrie B. Epidemiology of multimorbidity and implications for health care, research, and medical education: a cross-sectional study. The Lancet. 2012;380(9836):37-43\%U https://linkinghub.elsevier.com/retrieve/pii/S0140673612602402.

2. Boyd CM, Fortin M. Future of Multimorbidity Research: How Should Understanding of Multimorbidity Inform Health System Design? Public Health Reviews. 2010;32(2):451-74 \%U http://publichealthreviews.biomedcentral.com/articles/10.1007/BF03391611.

3. Feely A, Lix LM, Reimer K. Estimating multimorbidity prevalence with the Canadian Chronic Disease Surveillance System. Health Promot Chronic Dis Prev Can. 2017;37(7):215-22.

4. Koné Pefoyo AJ, Bronskill SE, Gruneir A, Calzavara A, Thavorn K, Petrosyan Y, et al. The increasing burden and complexity of multimorbidity. BMC Public Health. 2015;15(1 \%U http://bmcpublichealth.biomedcentral.com/articles/10.1186/s12889-015-1733-2).

5. St John PD, Tyas SL, Menec V, Tate R. Multimorbidity, disability, and mortality in community-dwelling older adults. Canadian Family Physician Medecin De Famille Canadien. 2014;60(5):e272-80.

6. Salako O, Okediji PT, Habeebu MY, Fatiregun OA, Awofeso OM, Okunade KS, et al. The pattern of comorbidities in cancer patients in Lagos, South-Western Nigeria. Ecancermedicalscience. 2018;12:843.

7. Leach CR, Weaver KE, Aziz NM, Alfano CM, Bellizzi KM, Kent EE, et al. The complex health profile of long-term cancer survivors: prevalence and predictors of comorbid conditions. J Cancer Surviv. 2015;9(2):239-51.

8. Ng HS, Roder D, Vitry A. Prevalence of Comorbidities and Health Status among Current Cancer and Cancer Survivors: An Australian Population-Based Study.: Value in Health; 2016.

9. Glynn LG, Valderas JM, Healy P, Burke E, Newell J, Gillespie P, et al. The prevalence of multimorbidity in primary care and its effect on health care utilization and cost. Fam Pract. 2011;28(5):516-23.

10. Tinetti ME, Fried TR, Boyd CM. Designing health care for the most common chronic condition-multimorbidity. JAMA. 2012;307(23):2493-4.

11. Fowler H, Belot A, Ellis L, Maringe C, Luque-Fernandez MA, Njagi EN, et al. Comorbidity prevalence among cancer patients: a population-based cohort study of four cancers. BMC Cancer. 2020;20(1):2.

12. Boakye D, Rillmann B, Walter V, Jansen L, Hoffmeister M, Brenner H. Impact of comorbidity and frailty on prognosis in colorectal cancer patients: A systematic review and meta-analysis. Cancer Treat Rev. 2018;64:30-9.

13. Ayeni OA, Norris SA, Joffe M, Cubasch H, Nietz S, Buccimazza I, et al. The multimorbidity profile of South African women newly diagnosed with breast cancer. Int J Cancer. 2020;147(2):361-74.

14. Gross CP, Guo Z, McAvay GJ, Allore HG, Young M, Tinetti ME. Multimorbidity and survival in older persons with colorectal cancer. J Am Geriatr Soc. 2006;54(12):1898-904.

15. Stairmand J, Signal L, Sarfati D, Jackson C, Batten L, Holdaway M, et al. Consideration of comorbidity in treatment decision making in multidisciplinary cancer team meetings: a systematic review. Ann Oncol. 2015;26(7):1325-32.

16. Gurney J, Sarfati D, Stanley J. The impact of patient comorbidity on cancer stage at diagnosis. Br J Cancer. 2015;113(9):1375-80.

17. Dima S, Chen KH, Wang KJ, Wang KM, Teng NC. Effect of Comorbidity on Lung Cancer Diagnosis Timing and Mortality: A Nationwide Population-Based Cohort Study in Taiwan. Biomed Res Int. 2018;2018:1252897.

18. Ritchie CS, Kvale E, Fisch MJ. Multimorbidity: an issue of growing importance for oncologists. J Oncol Pract. 2011;7(6):371-4.

19. Sharma M, Loh KP, Nightingale G, Mohile SG, Holmes HM. Polypharmacy and potentially inappropriate medication use in geriatric oncology. J Geriatr Oncol. 2016;7(5):346-53.

20. Morio K, Maeda I, Yokota I, Niki K, Murata T, Matsumura Y, et al. Risk Factors for Polypharmacy in Elderly Patients With Cancer Pain. Am J Hosp Palliat Care. 2019;36(7):598-602.

21. Chen RC, Royce TJ, Extermann M, Reeve BB. Impact of age and comorbidity on treatment and outcomes in elderly cancer patients. Semin Radiat Oncol. 2012;22(4):265-71.

22. Janssen-Heijnen ML, Maas HA, Houterman S, Lemmens VE, Rutten HJ, Coebergh JW. Comorbidity in older surgical cancer patients: influence on patient care and outcome. Eur J Cancer. 2007;43(15):2179-93.

23. Søgaard M, Thomsen RW, Bossen KS, Sørensen HT, Nørgaard M. The impact of comorbidity on cancer survival: a review. Clin Epidemiol. 2013;5(Suppl 1):3-29.

24. Gross CP, McAvay GJ, Guo Z, Tinetti ME. The impact of chronic illnesses on the use and effectiveness of adjuvant chemotherapy for colon cancer. Cancer. 2007;109(12):2410-9. 
25. Wojciechowska J, Krajewski W, Bolanowski M, Kręcicki T, Zatoński T. Diabetes and Cancer: a Review of Current Knowledge. Exp Clin Endocrinol Diabetes. 2016;124(5):263-75.

26. Gironés R, Torregrosa D, Gómez-Codina J, Maestu I, Tenias JM, Rosell R. Lung cancer chemotherapy decisions in older patients: the role of patient preference and interactions with physicians. Clin Transl Oncol. 2012;14(3):183-9.

27. Wolff JL, Starfield B, Anderson G. Prevalence, expenditures, and complications of multiple chronic conditions in the elderly. Arch Intern Med. 2002;162(20):2269-76.

28. Koroukian SM, Bakaki PM, Schluchter MD, Owusu C. Treatment and survival patterns in relation to multimorbidity in patients with locoregional breast and colorectal cancer. J Geriatr Oncol. 2011;2(3):200-8.

29. Rubin G, Berendsen A, Crawford SM, Dommett R, Earle C, Emery J, et al. The expanding role of primary care in cancer control. Lancet Oncol. 2015;16(12):1231-72.

30. Piette JD, Kerr EA. The impact of comorbid chronic conditions on diabetes care. Diabetes Care. 2006;29(3):725-31.

31. Wallace E, Salisbury C, Guthrie B, Lewis C, Fahey T, Smith SM. Managing patients with multimorbidity in primary care. BMJ. 2015;350:h176.

32. Lee S, Rothbard A, Choi S. Effects of comorbid health conditions on healthcare expenditures among people with severe mental illness. J Ment Health. 2016;25(4):291-6.

33. Coleman EA. Falling through the cracks: challenges and opportunities for improving transitional care for persons with continuous complex care needs. $J$ Am Geriatr Soc. 2003;51(4):549-55.

34. Sussman J, Baldwin LM. The interface of primary and oncology specialty care: from diagnosis through primary treatment. J Natl Cancer Inst Monogr. 2010;2010(40):18-24.

35. Del Giudice ME, Grunfeld E, Harvey BJ, Piliotis E, Verma S. Primary care physicians' views of routine follow-up care of cancer survivors. J Clin Oncol. 2009;27(20):3338-45.

36. Potosky AL, Han PK, Rowland J, Klabunde CN, Smith T, Aziz N, et al. Differences between primary care physicians' and oncologists' knowledge, attitudes and practices regarding the care of cancer survivors. J Gen Intern Med. 2011;26(12):1403-10.

37. Diederichs C, Berger K, Bartels DB. The measurement of multiple chronic diseases-a systematic review on existing multimorbidity indices. J Gerontol A Biol Sci Med Sci. 2011;66(3):301-11.

38. Fortin M, Stewart M, Poitras ME, Almirall J, Maddocks H. A systematic review of prevalence studies on multimorbidity: toward a more uniform methodology. Ann Fam Med. 2012;10(2):142-51.

39. Katikireddi SV, Skivington K, Leyland AH, Hunt K, Mercer SW. The contribution of risk factors to socioeconomic inequalities in multimorbidity across the lifecourse: a longitudinal analysis of the Twenty-07 cohort. BMC Medicine. 2017;15(1 \% U http://bmcmedicine.biomedcentral.com/articles/10.1186/s12916-017-0913-6).

40. Canizares M, Hogg-Johnson S, Gignac MAM, Glazier RH, Badley EM. Increasing Trajectories of Multimorbidity Over Time: Birth Cohort Differences and the Role of Changes in Obesity and Income. The Journals of Gerontology: Series B. 2018;73(7):1303-14 \% U https://academic.oup.com/psychsocgerontology/article/73/7//2990202.

41. Ontario CC. Cervical Screening [Available from: https://www.cancercareontario.ca/en/types-ofcancer/cervical/screening\#: :text=When\%20to\%20Get\%20Screened.\%20The\%200ntario\%20Cervical\%20Screening,by\%20a\%20doctor\%20or\%20nurse\%2C

42. Seigneurin A, Delafosse P, Trétarre B, Woronoff AS, Velten M, Grosclaude P, et al. Are comorbidities associated with long-term survival of lung cancer? A population-based cohort study from French cancer registries. BMC Cancer. 2018;18(1):1091.

43. Legler A, Bradley EH, Carlson MD. The effect of comorbidity burden on health care utilization for patients with cancer using hospice. J Palliat Med. 2011;14(6):751-6.

44. Druss BG, Marcus SC, Olfson M, Pincus HA. The most expensive medical conditions in America. Health Aff (Millwood). 2002;21(4):105-11.

45. Muggah E, Graves E, Bennett C, Manuel DG. Ascertainment of chronic diseases using population health data: a comparison of health administrative data and patient self-report. BMC public health. 2013;13(1):16.

46. Gruneir A, Griffith LE, Fisher K, Perez R, Favotto L, Patterson C, et al. Measuring multimorbidity series. An overlooked complexity - Comparison of selfreport vs. administrative data in community-living adults: Paper 3 . Agreement across data sources and implications for estimating associations with health service use. J Clin Epidemiol. 2020;124:173-82.

47. Verheij RA, Curcin V, Delaney BC, McGilchrist MM. Possible Sources of Bias in Primary Care Electronic Health Record Data Use and Reuse. J Med Internet Res. 2018;20(5):e185.

48. Davis KA, Sudlow CL, Hotopf M. Can mental health diagnoses in administrative data be used for research? A systematic review of the accuracy of routinely collected diagnoses. BMC Psychiatry. 2016;16:263.

49. Vin-Raviv N, Akinyemiju TF, Galea S, Bovbjerg DH. Depression and Anxiety Disorders among Hospitalized Women with Breast Cancer. PLoS One. 2015;10(6):e0129169.

50. Terret C, Castel-Kremer E, Albrand G, Droz JP. Effects of comorbidity on screening and early diagnosis of cancer in elderly people. Lancet Oncol. 2009;10(1):80-7.

51. Stewart M, Fortin M, Team* P-ClfPwM. Patient-Centred Innovations for Persons with Multimorbidity: funded evaluation protocol. CMAJ Open. 2017;5(2):E365-E72.

\section{Figures}




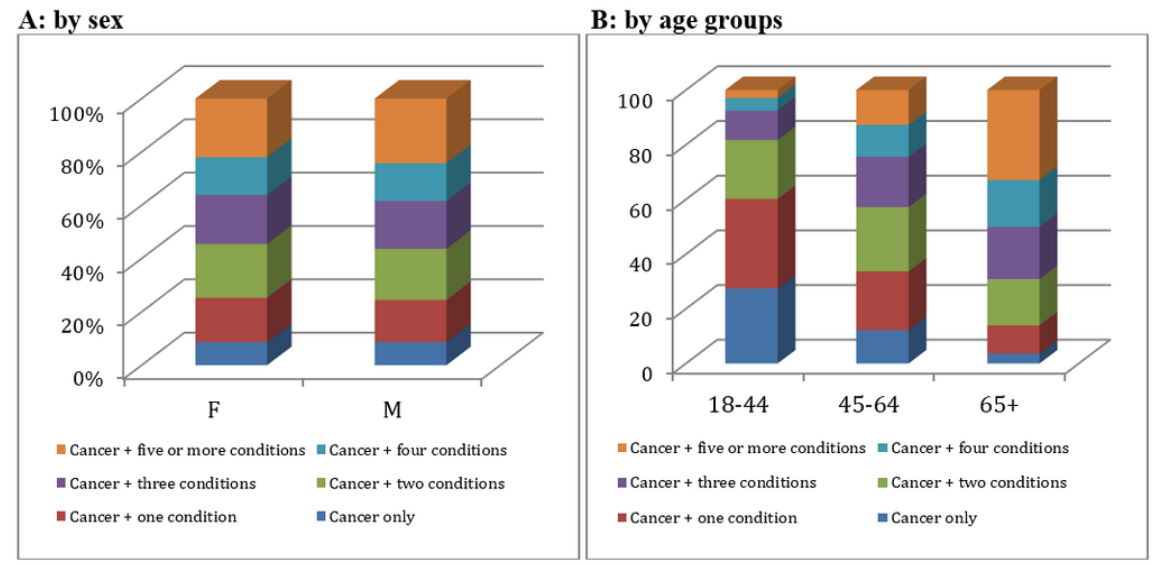

C: by cancer types

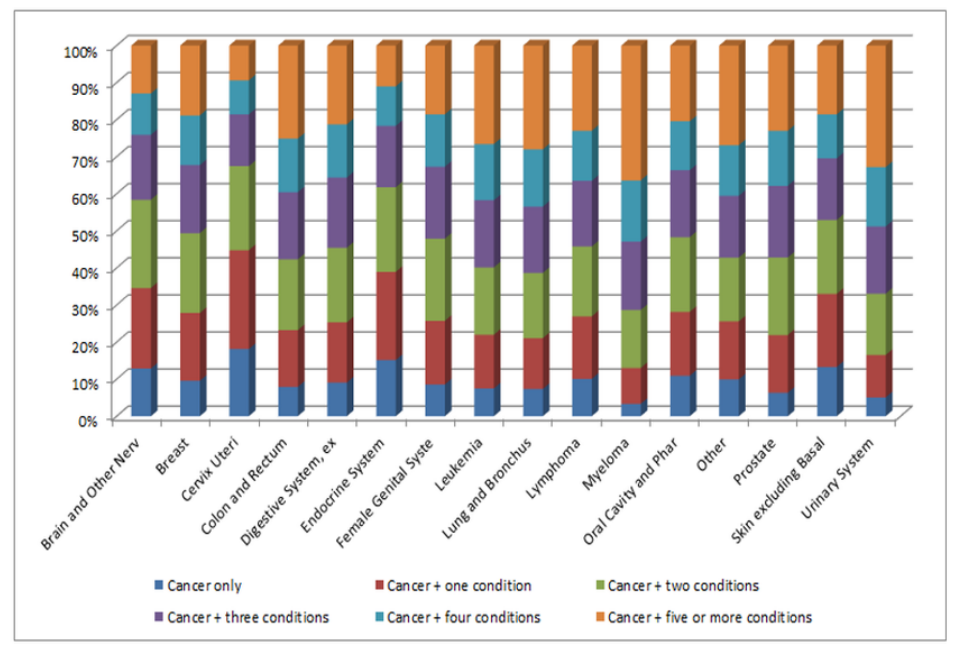

Figure 1

MMB level by sex, age, and cancer site 


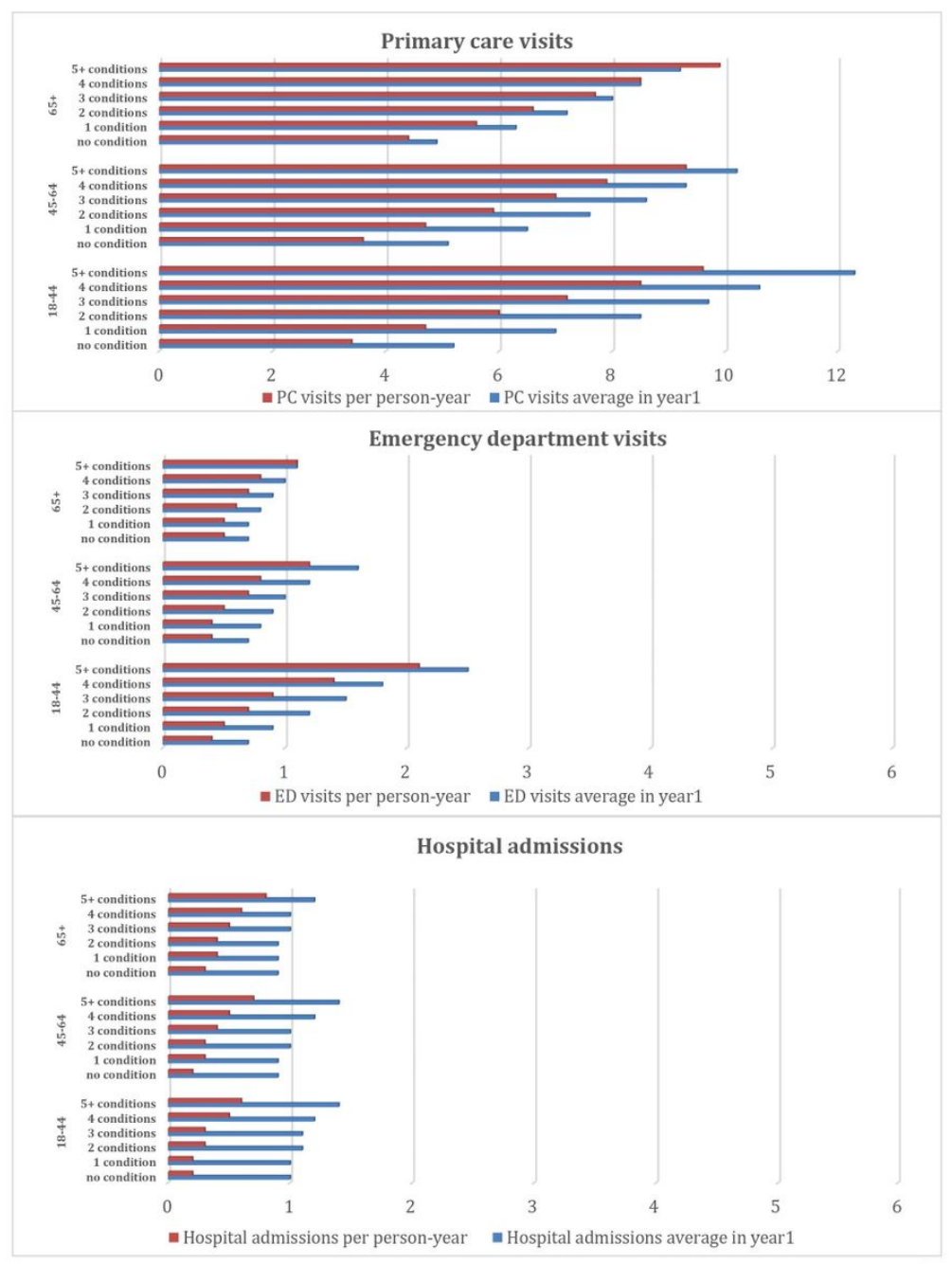

\section{Figure 2}

Health Service Utilization (HSU) by MMB level, prior to and following cancer diagnosis, by age, among those with at least 30 days of follow-up ( $\mathrm{n}=560,570$ ) 


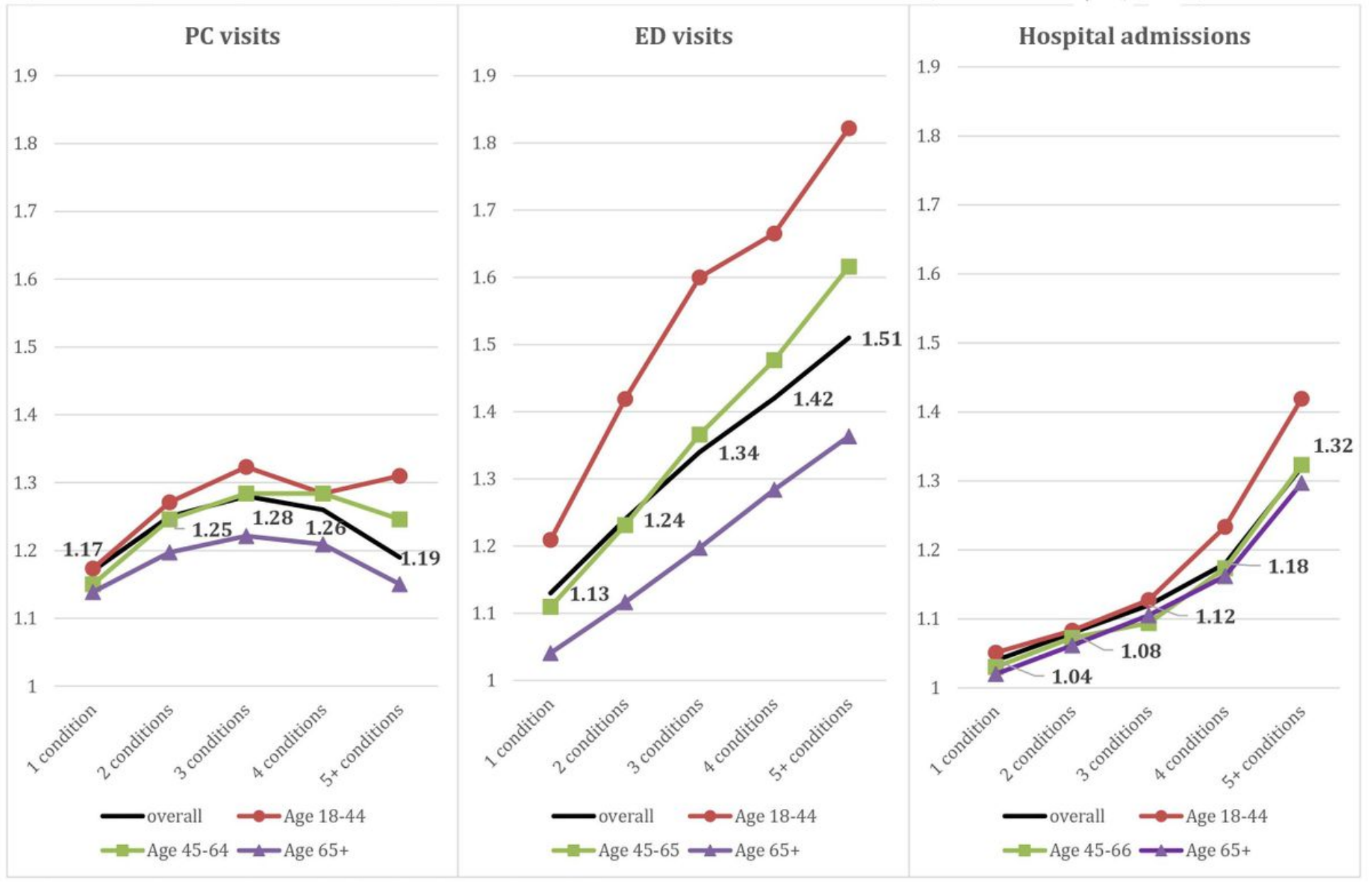

Figure 3

Adjusted impact of MMB (IRR) on the number of health services encounters, overall and by age groups Incidence rate ratio (IRR) values are displayed for the overall population

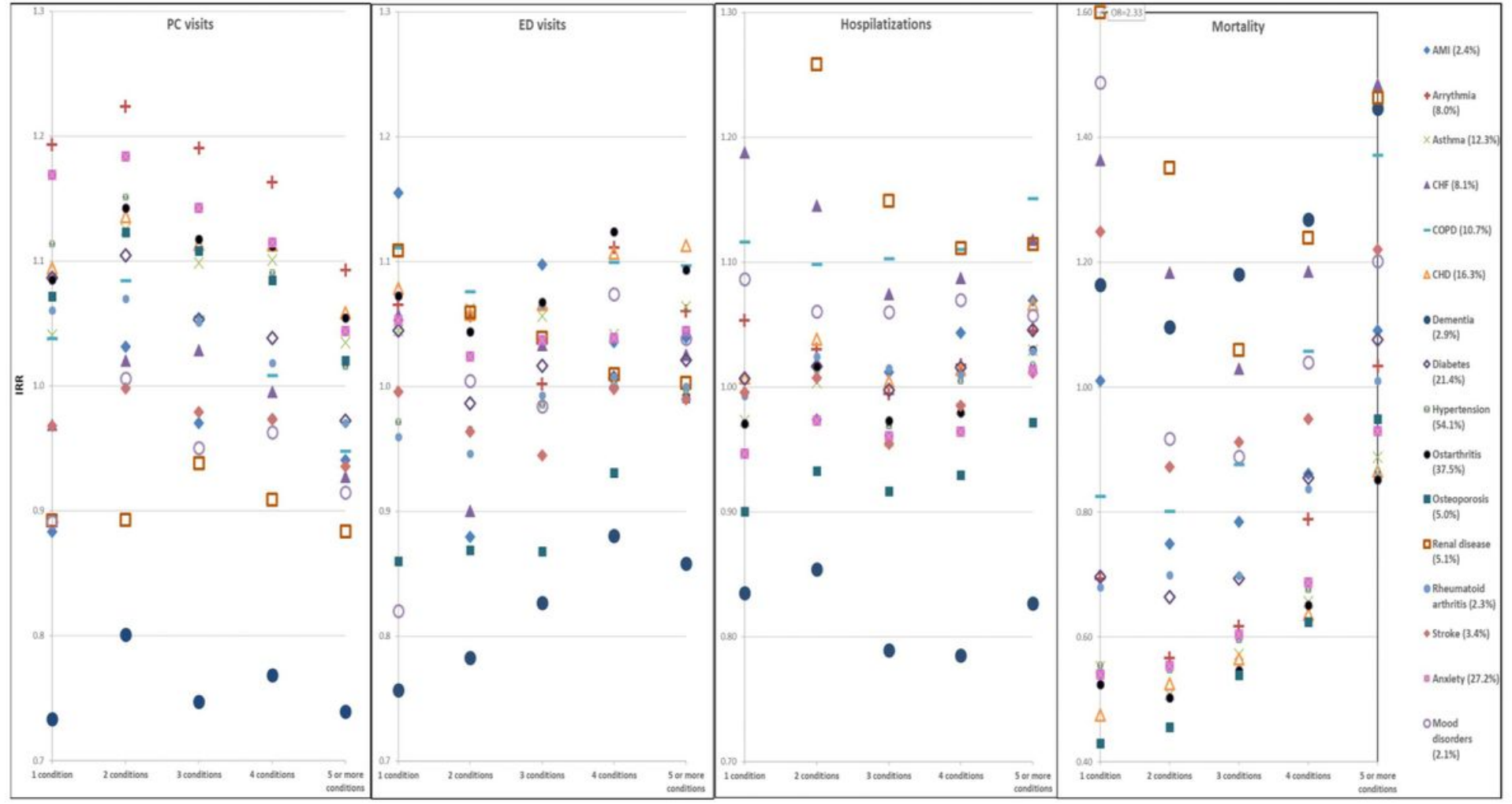


Figure 4

Incidence rate ratio (IRR) shows the impact of pre-existing individual conditions on health service use and mortality, by MMB levels Prevalence of each condition in the study population is provided between bracket. For example, $5.1 \%$ of those who survived at least 30 days had been diagnosed with renal disease prior to cancer; $54.1 \%$ with hypertension, etc. As shown, impact of each condition greatly varies by MMB level, and between conditions within the same level.
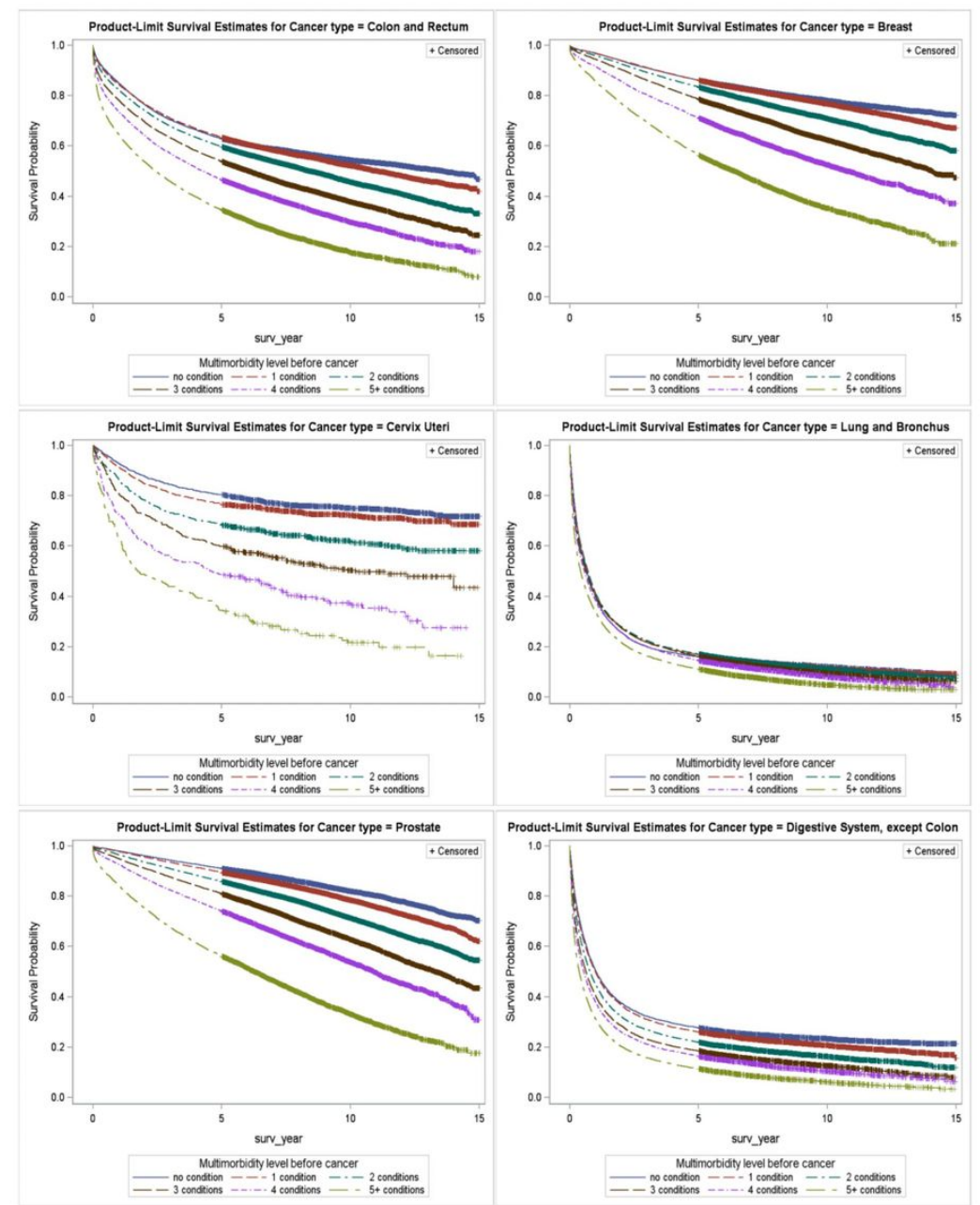

\section{Figure 5}

Survival curve by MMB for selected cancer types

\section{Supplementary Files}

This is a list of supplementary files associated with this preprint. Click to download.

- Appendices.docx 